\title{
Terahertz Circular Dichroism Spectroscopy of Biomaterials Enabled by Kirigami Polarization Modulators
}

\author{
Won Jin Choi $^{1 \dagger}$, Gong Cheng ${ }^{2,3 \dagger}$, Zhengyu Huang ${ }^{4}$, Shuai Zhang 5 , \\ Theodore B. Norris ${ }^{3,4^{*}}$ and Nicholas A. Kotov ${ }^{1,6,7,8^{*}}$
}

${ }^{1}$ Department of Materials Science and Engineering, University of Michigan, Ann Arbor, Michigan 48109, USA;

${ }^{2}$ Department of Physics, University of Michigan, Ann Arbor, Michigan 48109, USA;

${ }^{3}$ Center for Ultrafast Optical Science, University of Michigan, Ann Arbor, Michigan 48109, USA;

${ }^{4}$ Department of Electrical Engineering and Computer Science, University of Michigan, Ann Arbor, Michigan 48109, USA;

${ }^{5}$ Taubman College of Architecture and Urban Planning, University of Michigan, Ann Arbor, Michigan 48109, USA;

${ }^{6}$ Department of Chemical Engineering, University of Michigan, Ann Arbor, Michigan 48109, USA;

${ }^{7}$ Program in Macromolecular Science and Engineering, University of Michigan, Ann Arbor, Michigan 48109, USA;

${ }^{8}$ Biointerfaces Institute, University of Michigan, Ann Arbor, Michigan 48109, USA;

$\dagger$ Both authors contributed equally to this work. *To whom correspondence should be addressed.

E-mail: tnorris@umich.edu (T.B.N.), kotov@umich.edu (N.A.K.)

\begin{abstract}
S: Terahertz circular dichroism (TCD) offers spectroscopic capabilities for understanding mesoscale chiral architecture and low-energy vibrations of macromolecules in (bio)materials ${ }^{1-5}$. However, the lack of dynamic polarization modulators comparable to polarization optics for other parts of electromagnetic spectrum impedes proliferation of TCD spectroscopy $^{6-10}$. Here we show that tunable optical elements fabricated from patterned plasmonic sheets with periodic kirigami cuts make possible polarization modulation of THz radiation under application of mechanical strain. A herringbone pattern of microscale metal
\end{abstract}


stripes enables dynamic range of polarization rotation modulation exceeding $\mathbf{8 0}^{\circ}$ over thousands of cycles. Upon out-of-plane buckling, the plasmonic stripes function as reconfigurable semi-helices of variable pitch aligned along the $\mathrm{THz}$ propagation direction. Several biomaterials, exemplified by elytrons of Chrysina gloriosa beetles, revealed distinct TCD fingerprints associated with the helical substructure in the biocomposite. Analogous kirigami modulators will also enable other applications in THz optics, such as polarizationbased terahertz imaging and phase-encrypted telecommunication.

Scientific and technological advances related to chirality of liquid crystals, biomolecules, and synthetic drugs were enabled by the prior development of chiroptical spectroscopies, notably electronic circular dichroism (ECD) and vibrational circular dichroism (VCD), which enable identification of mirror asymmetry at molecular and nanometer scales. ECD and VCD are based on the modulation of circularly polarized light with photon energies in the ranges of $1.5-7 \mathrm{eV}$ and 0.07-0.5 eV, respectively, which limit the physical dimensions and the resonant energies of the chiral structures that can be probed. Of particular interest is the far infrared (IR) or terahertz (THz) region of the electromagnetic spectrum corresponding to wavelengths in the range $0.1-1$ $\mathrm{mm}$ and photon energies from $\sim 0.001 \mathrm{eV}$ to $\sim 0.01 \mathrm{eV}^{11-15}$ Besides being informative for many areas of $\mathrm{THz}$ studies from astronomy and solid-state physics to telecommunication, $\mathrm{THz}$ circular dichroism (TCD) is essential for understanding of biomaterials, biomolecules, and pharmaceuticals because the energy of THz photons enables probing the 'soft' oscillatory motions of biomolecules $^{1-5}$. The practical realization of TCD, however, has proven to be an elusive goal due to the difficulties with polarization modulation of $\mathrm{THz}$ radiation. The key problem is the lack of optical components for modulation of circular polarization in the $\mathrm{THz}$ regime, which is easily 
accomplished at shorter wavelengths using photoelastic modulators (PEM), half- and quarter waveplates, and lately with chiral metamaterials and metasurfaces ${ }^{6-9}$ Although the modulation of linearly and circularly polarized $\mathrm{THz}$ beams has been demonstrated with fairly complicated and bulky optical systems based on $\mathrm{THz}$ metamaterials, e.g. with pneumatic control of scattering elements ${ }^{6}$, sufficiently strong and dynamic polarization rotation of $\mathrm{THz}$ radiation remains a significant challenge ${ }^{6-10}$.

Kirigami, the oriental art of paper cutting, presents a powerful tool to create complex and tunable three-dimensional (3D) geometries from simple (2D) two-dimensional cut patterns, which can be scaled across many orders of magnitude to yield macro to nanoscale structures ${ }^{16-22}$. The ability to achieve out-of-plane deformations and designed 3D shapes, the robustness of the patterns under cyclic reconfiguration and the manufacturing simplicity of kirigami structures together promise untapped possibilities for the efficient modulation of $\mathrm{THz}$ optical beams. Here we show that kirigami optics affords real-time modulation of $\mathrm{THz}$ beams with polarization rotation and ellipticity angles as large as $80^{\circ}$ and $40^{\circ}$ over thousands of cycles, respectively. The unusually large amplitudes of polarization rotation and ellipticity angles were enabled by double-scale patterns comprised of microscale metallic stripes together with wavelength-scale kirigami cuts.

The kirigami modulators in this study are made from parylene - a stiff polymer (Young's modulus $E=2.8 \mathrm{GPa}$ ) with high transparency across the $\mathrm{THz}$ spectrum ${ }^{23}$. Parylene sheets were patterned with straight cuts in a face centered rectangular lattice with a periodicity of $p_{c u t}=600$ $\mu \mathrm{m}$ (Supplementary Fig. 1 and 2). This 2D pattern transforms upon stretching into an array of alternating convex and concave out-of-plane surfaces due to buckling. (Fig. 1a and Supplementary Fig. 4). 
The function-defining structural feature was gold herringbone pattern with $D_{n}$ symmetry deposited on parylene sheets in registry with kirigami cuts. When buckled, the patterned surface is transformed into a homeomorph of a three-dimensional helix (Fig. 1). Its pitch varies under mechanical strain while its long axis remains aligned with the surface normal (z-axis) and with propagating $\mathrm{THz}$ beam. Unlike microfabricated metallic helices ${ }^{19,24}$ and semi-dimensional metaoptics ${ }^{25,26}$, the double-patterns kirigami surfaces enable strong and tunable polarization rotation with real time modulation. Their confocal microscopy images (Supplementary Figs 4 and 5) obtained for strains $\varepsilon$ from $0 \%$ to $22.5 \%$ demonstrated that buckling and tilting of each outof-plane segment occurred simultaneously for the entire sheet, which is essential for uniform polarization front of a beam. The reconstructed contour maps of left-handed $\left(L_{-}\right)$and right-handed $(R-)$ kirigami structures (Fig. 2b, Supplementary Fig. 4) at $\varepsilon=22.5 \%$ strain indicate that the edges of the buckled elements extended to $68 \mu \mathrm{m}$ symmetrically along the positive and negative z-axis, tuning the radius and pitch of the clockwise and counter clockwise half-helices ${ }^{19,24}$ are the two key factors for controlling electrodynamic interactions of these structures with left- and right circularly polarized photons. The experimental deformations matched the predictions from finiteelement modeling exactly (Supplementary Fig. 4c and 4d).

$\mathrm{THz}$ time-domain spectroscopy (THz-TDS) over the range 0.2-2 $\mathrm{THz}$ was used to characterize chiroptical performance of the kirigami modulators. The polarization rotation angle, $\theta$, and ellipticity angle $\eta$ (Supplementary Information) of the $\mathrm{THz}$ beam after passing through kirigami sheets or biomaterials with expected TCD activity were determined using two complementary methods. The first protocol was based on standard calculations of Stokes parameters from the Jones matrix from a sequence of linear polarization measurements; the second one was based on direct measurements employing kirigami modulators. 
The kirigami sheets were mounted on an optical holder and finely controllable stress was applied with a programmable piezoactuator with a precision of $100 \mathrm{~nm}(\varepsilon=0.001 \%)$ (Fig. 2a). As expected $\theta$ and $\eta$ increased with strain and kirigami structures with left-handed and right-handed herringbone patterns exhibit THz responses that are nearly identical but with opposite signs (Fig. $2 \mathrm{c}-2 \mathrm{~h}$ ). The inclination angle $(\varphi)$ of the herringbone patterns (insets of Fig. $2 \mathrm{~d}, \mathrm{f}, \mathrm{h}$ ) determined the position of the main resonance peaks, which were observed at $0.41 \mathrm{THz}$ for $\varphi=15^{\circ}, 0.62 \mathrm{THz}$ for $\varphi=30^{\circ}$ and $0.81 \mathrm{THz}$ for $\varphi=45^{\circ}$. The maximum values of $\theta$ and $\eta$ reached as high as $80^{\circ}$ and $40^{\circ}$, respectively, were obtained for herringbone patterns with $\varphi$ of $30^{\circ}$. This maximum ellipticity value is almost close to that of quarter-waveplate. Note that the magnitudes of $\theta$ and $\eta$ can be different depending on the in-plane rotation angles due to birefringence, which was taken into account in the TCD spectra (Supplementary Figs 8-11). As a control, an achiral pattern with horizontally aligned $\left(\varphi=0^{\circ}\right)$ Au strips was tested, and showed near-zero values of $\theta$ and $\eta$ regardless of the strain, confirming the critical role of the double-pattern design for the strong optical activity (Supplementary Fig. 11). Polarization modulations with nearly identical values of $\theta$ and $\eta$ were obtained for 1000 cycles with $\varepsilon$ between $0 \%$ and $22.5 \%$ (Supplementary Fig. 12).

The effect of the microscale cut pattern on the optical performance of the kirigami polarization modulators was tested for variable size of the unit cell for a constant inclination $\varphi=$ $30^{\circ}$. As $L_{c u t}$ becomes larger, the main TCD peak shifts to the red (Fig. $3 \mathrm{a}$ and $3 \mathrm{~b}$ ), indicating that its spectral position is determined by the longitudinal plasmonic resonances of the Au strips. Confirming this conclusion, TCD spectra of kirigami modulators with Au strips having same length and $\varphi$ but narrower width display the same position of resonance peak and similar overall shape (Supplementary Fig. 11). The resonance wavelength of the kirigami sheets with herringbone patterns can be heuristically assessed as an $L C$ circuit with the resonance frequency of $f_{r}=$ 
$\left(2 \pi \sqrt{L_{A u} C_{A u}}\right)^{-1} 27$. The inductance $L_{A u}$ and capacitance $C_{A u}$ of Au strips scale linearly with its length, $l$ (Fig. 3c, Supporting Information), and therefore $f_{r}$ becomes inversely proportional to $l$ (Fig. 3d). Alternatively, the metal strips can also be approximated as Hertzian dipoles bent and tilted in 3D space, $l \sim \lambda_{r} / 4=c / 4 f_{r}$, where $c$ and $\lambda_{r}$ are the speed of light and resonance wavelength. This equation can be used to provide an approximate guide of the design of herringbone patterns for different applications.

TCD spectra of the kirigami optical components can be predicted with $a b$ ovo electrodynamic simulations. Computed TCD spectra (Fig. 4a) matched well the experimental data with respect to the signs of the polarization rotation angle, peak positions, relative peak widths and amplitudes (Supplementary Fig. 11a). Calculated time-averaged current norm distributions generated on the Au strips for the incident of the circularly polarized beam point to the origin of the plasmonic states responsible for individual peaks (Fig. 4b-d) ${ }^{6}$. At the off-resonant frequency of $0.57 \mathrm{THz}$, the induced currents are low for both co- and cross-circularly polarized beam and most of the Au strips are optically inactive (Fig. 4b). At the resonant frequency of $0.82 \mathrm{THz}$, however, the incident beam induces strong currents in the Au strips. Simultaneously, the currents excited by the right-handed circularly polarized beam significantly exceed those for left circularly polarized beam (Fig. 4c and 4d). As a result, the transmittance of the left circularly polarized beam is larger than that of the right circularly polarized beam due to the induced current, which is consistent with the sign of the peaks in Fig. 4a and Supplementary Fig. 11a.

The unique combination of high ellipticity and tunability of kirigami optics makes possible its utilization of for modulating THz light beams in practical realizations of TCD spectroscopy to investigate biological and other materials that are opaque in the visible range but transparent for THz radiation. To demonstrate this capability, we measured TCD spectra of several representative 
biological samples (Fig. 5a and Supplementary Fig. 22), including a leaf of sugar maple tree (Acer saccharum), an elytron of green beetle (Chrysina gloriosa), a petal of dandelion (Taraxacum officinale) and a piece of pig fat. Here, TCD spectra were calculated directly from difference of transmission-intensity between left and right elliptically polarized $\mathrm{THz}$ beam (EPB) generated by kirigami modulators according to Eq. 1:

$$
T C D \text { by kirigami modulator }=\tan ^{-1}\left(\frac{I_{L K}^{1 / 2}-I_{R K}^{1 / 2}}{I_{L K}^{1 / 2}+I_{R K}^{1 / 2}}\right)
$$

where, $I_{L K}$ and $I_{R K}$ are the intensities of the left and right EPB after passing through sample, respectively. We observed distinct $\mathrm{THz}$ spectra from the tested biomaterials that can be associated with low energy vibrational modes of their biological components and with chiral structural organization. In both cases, the opaqueness of biomaterial and mismatch in energy/wavelengths with visible light would not allow chiral characterization by ECD or VCD spectroscopy. An exemplary case is the transmissive TCD measurements of an elytron of C. gloriosa beetle (Fig.5b and c), which is known to have the selective reflection of circularly polarized light in the visible range ${ }^{28}$. A positive peak of TCD (Fig.5g) as large as about $3^{\circ}$ at $0.68 \mathrm{THz}$ is observed in the red circled area in Fig.5f. Notably, the absorption peak of this biological composite (Fig.5h) is well aligned with that of TCD peak. This TCD spectrum is associated with the chirality of its micro structure of exocuticle (Fig. 5d) which shows chiroptical response in reflected light in visible range (Fig. 5e $)^{28}$.

In conclusion, the double-pattern design of kirigami materials combining submillimeter cuts and nanometer scale plasmonic stripes affords the real-time tunability of helical structures oriented perpendicularly to the propagation of the light beam. Kirigami optical elements make possible realization of TCD spectroscopy and better understanding of liquid-crystal-like 
organization of soft and mineralized tissues ${ }^{29,30}$. The lightweight capabilities and high polarization efficiency of kirigami optics open a possibility of portable compact $\mathrm{THz}$ spectrometers. The realization of real-time polarization modulation of $\mathrm{THz}$ beams also enable advances in secure high bandwidth communication and non-invasive imaging. 




Kirigami substrate

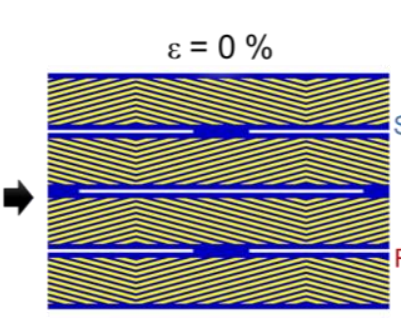

Herringbone patterned Kirigami substrate

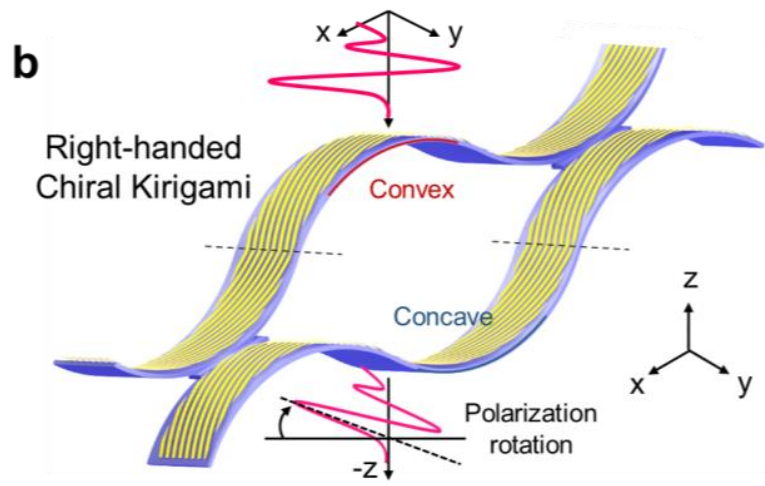

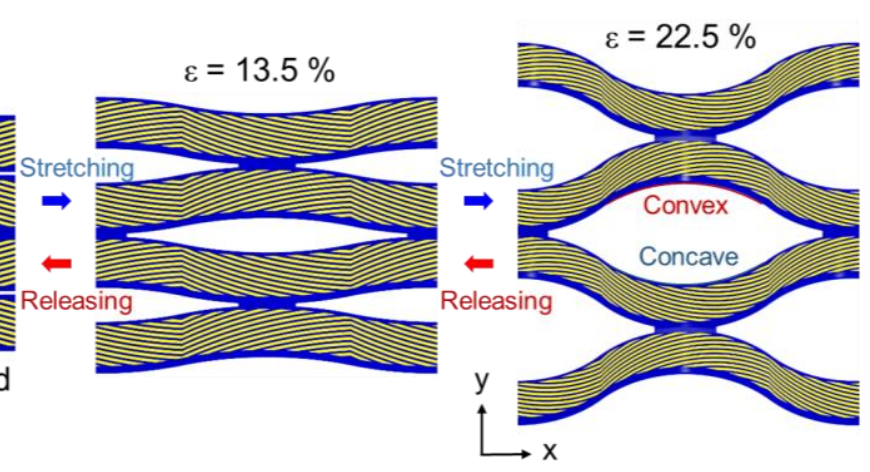

C

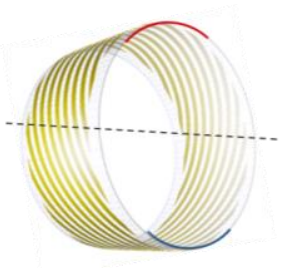

Right-handed Helix d

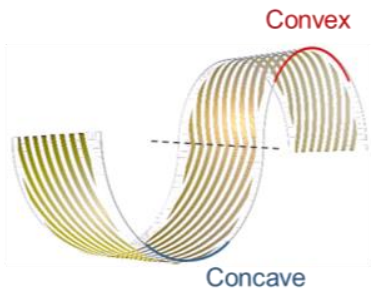

Homeomorphic subsets of Helix

Figure 1. Schematic of chiral kirigami topology. a, Herringbone structured Au strips are deposited on the kirigami substrate. This chiral kirigami topology can tune the polarization rotation angle and ellipticity by mechanical force. b, Stretched chiral kirigami metamaterial that is topologically equivalent helix structure. c, Standard right-handed helix structure whose outside is covered with slanted striations and the structure which has homeomorphic subsets of helix. 
a
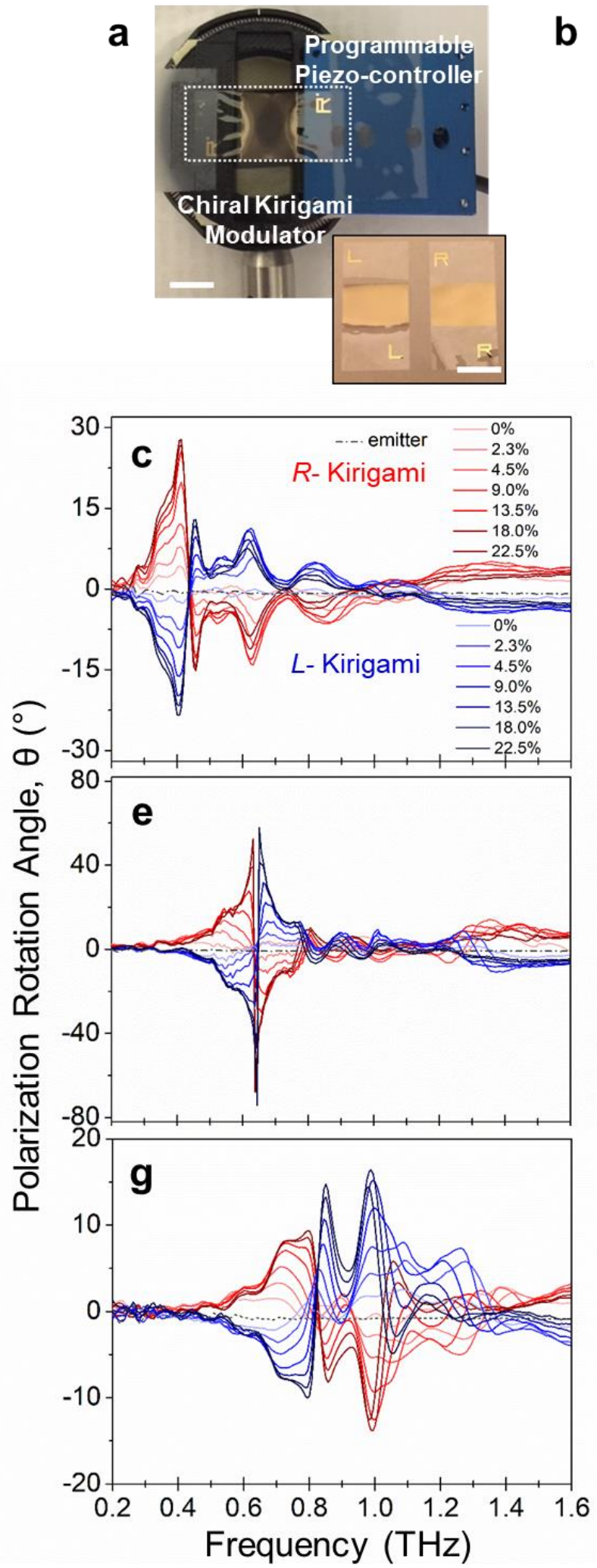

b
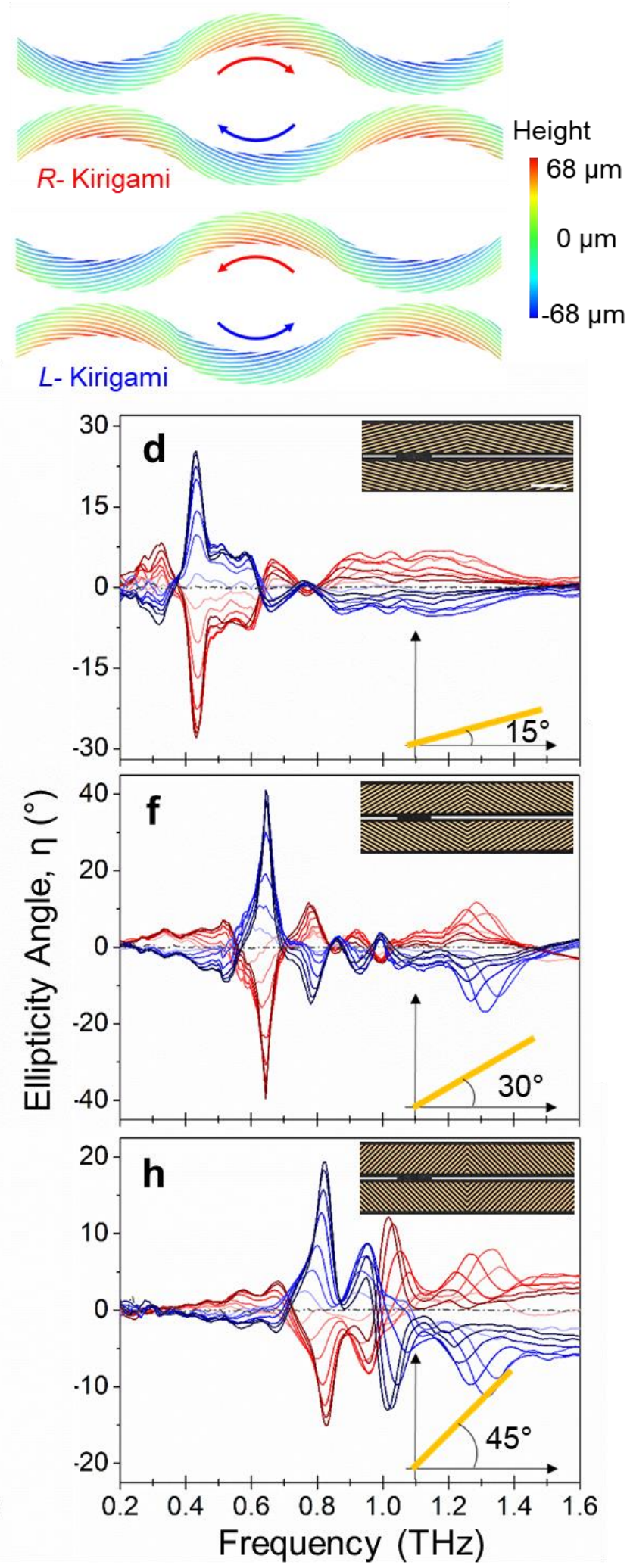
Figure 2. THz-TDS measurement of chiral kirigami modulator. a, kirigami mounted on the 3D printed rotatable optical holder with piezoelectric controller. Subset figure shows photo image of left and right handed chiral kirigami substrate. Yellow shiny region is the herringbone patterned $\mathrm{Au}$ zone. Both of scale bars are $1 \mathrm{~cm}$. b, Contour map of kirigami modulator reconstructed from laser confocal microscope images. $\mathbf{c}$, e and $\mathbf{g}$, Results of polarization rotation angle of kirigami having slanted $\mathrm{Au}$ angles $(\varphi)$ of 15, 30, 45 degree with respect to strain $(\%)$, respectively. d, f and $\mathbf{h}$, Results of consequent ellipticity angle of kirigami having slanted Au angles $(\varphi)$ of 15, 30, 45 degree with respect to various strain, respectively. One can notice that there is an approximately $0.2 \mathrm{THz}$ increment per $15^{\circ}$ change of $\varphi$. As can be expected by the Kramers-Kronig relation ${ }^{11}$, the ellipticity exhibited a dispersive curve and crossed zero at slightly off-resonance frequencies, where the polarization rotation showed maximum. Inset shows optical microscope images of each kirigami samples. Scale bar in $\mathbf{d}$ is $100 \mu \mathrm{m}$. 

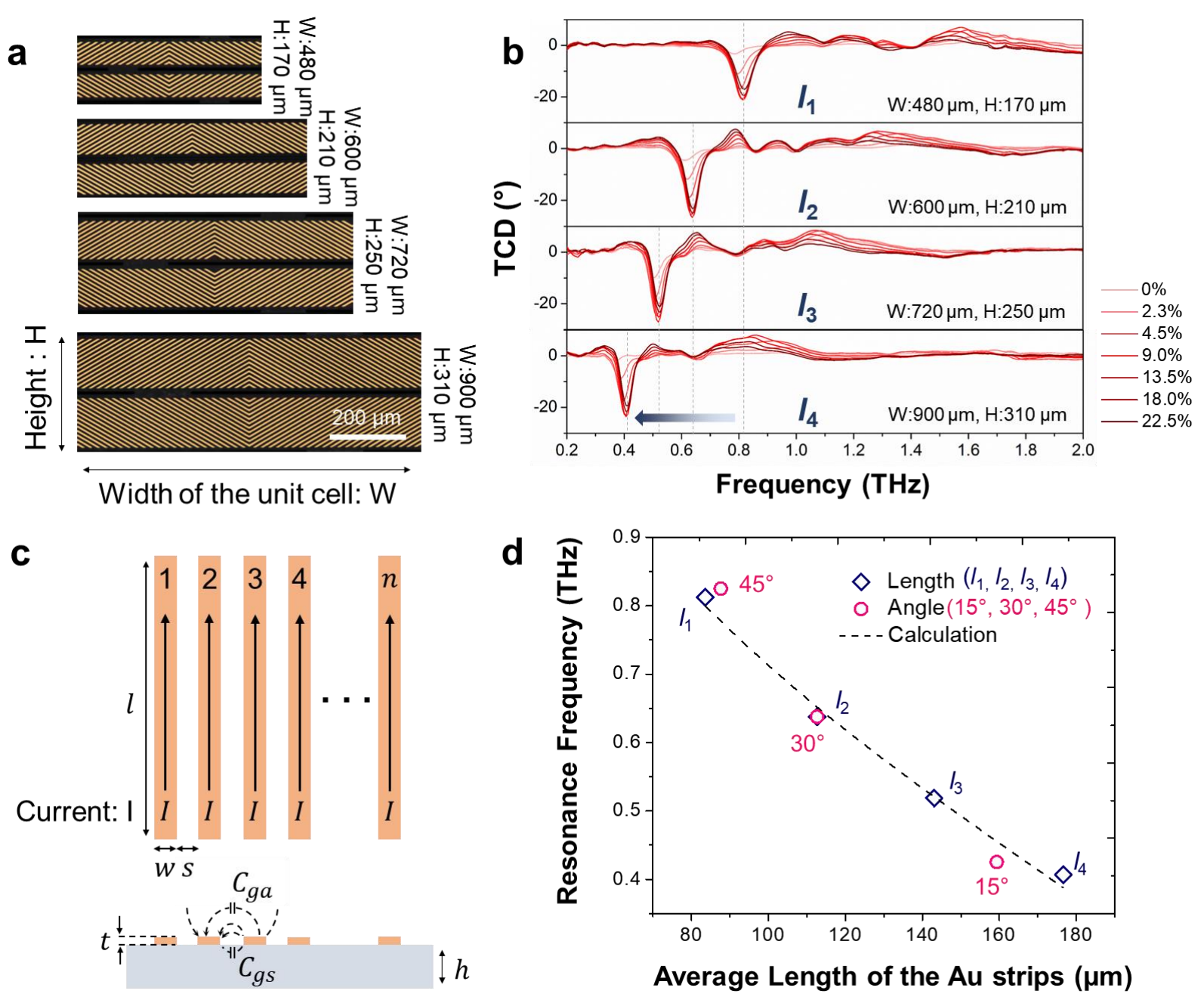

Average Length of the Au strips ( $\mu \mathrm{m})$

Figure 3. Understanding physical meaning of resonance frequency. a, Optical microscope image of various length of unit cell. All samples have $\varphi$ of $30^{\circ}$. $\mathbf{b}$, Result of measured TCD from $R$ - kirigami modulator with various length of unit cell. $\mathbf{c}$, Upper figure shows parallel conducting strips to obtain total inductance of this configuration. Lower figure is for calculating capacitance of array of strips. d, Relation between resonance frequency and average length $(l)$ of the Au strips. Scale bar in $\mathbf{a}$ is $200 \mu \mathrm{m}$. 

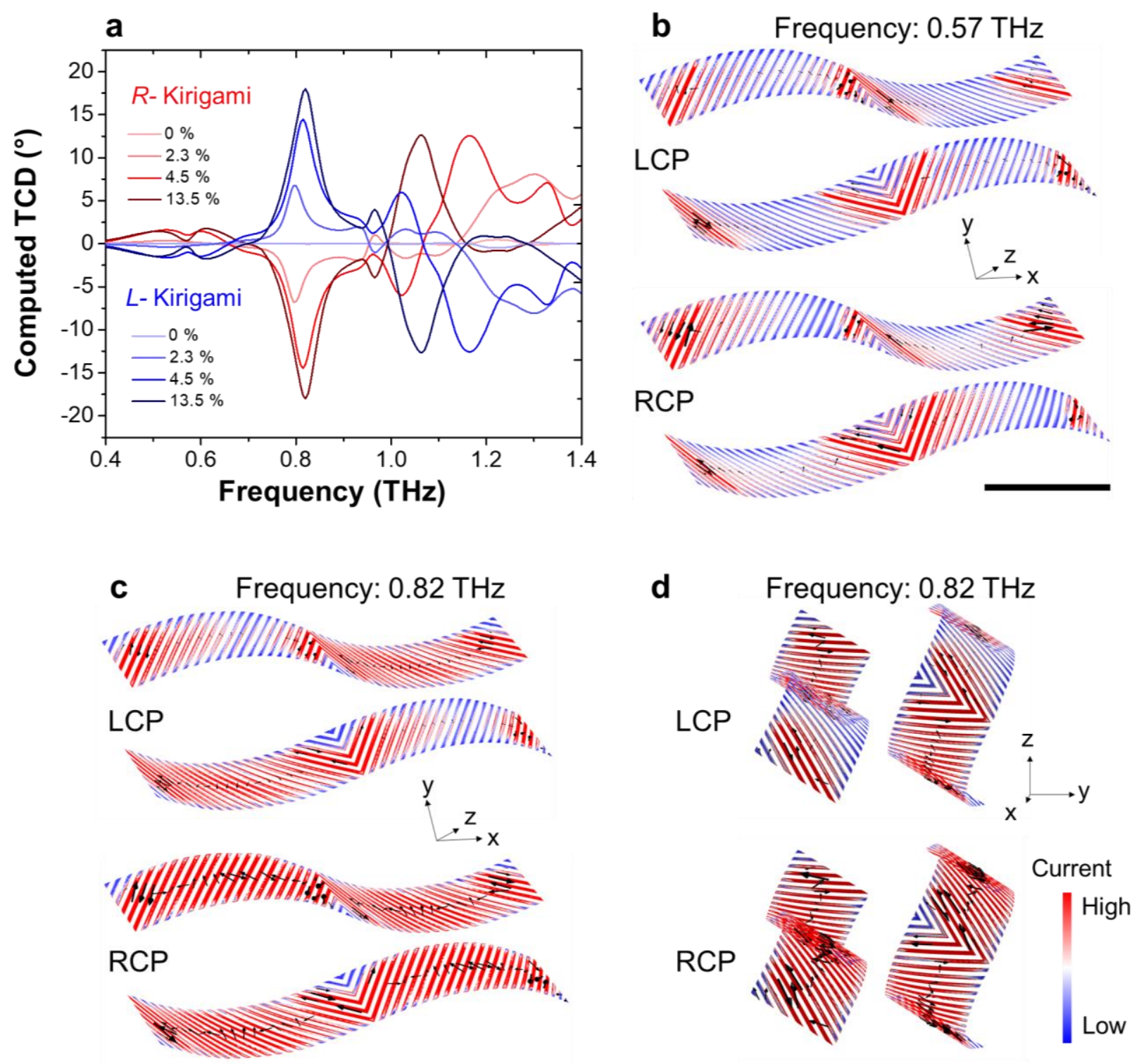

Figure 4. Computed terahertz circular dichroism and time-averaged current norm distributions on kirigami modulator $\left(\varphi=45^{\circ}\right)$. a, Computed TCD spectrum for 4 deformation states. $\mathbf{b}$ and $\mathbf{c}$, Tilted view of current norm distributions of $R$ - kirigami at the frequency of 0.57 $\mathrm{THz}, 0.82 \mathrm{THz}$, respectively. $\mathbf{d}$, Side view of current norm distributions of $R$ - kirigami at $0.82 \mathrm{THz}$. Black arrows indicate the current directions. A scale bar is $200 \mu \mathrm{m}$. 

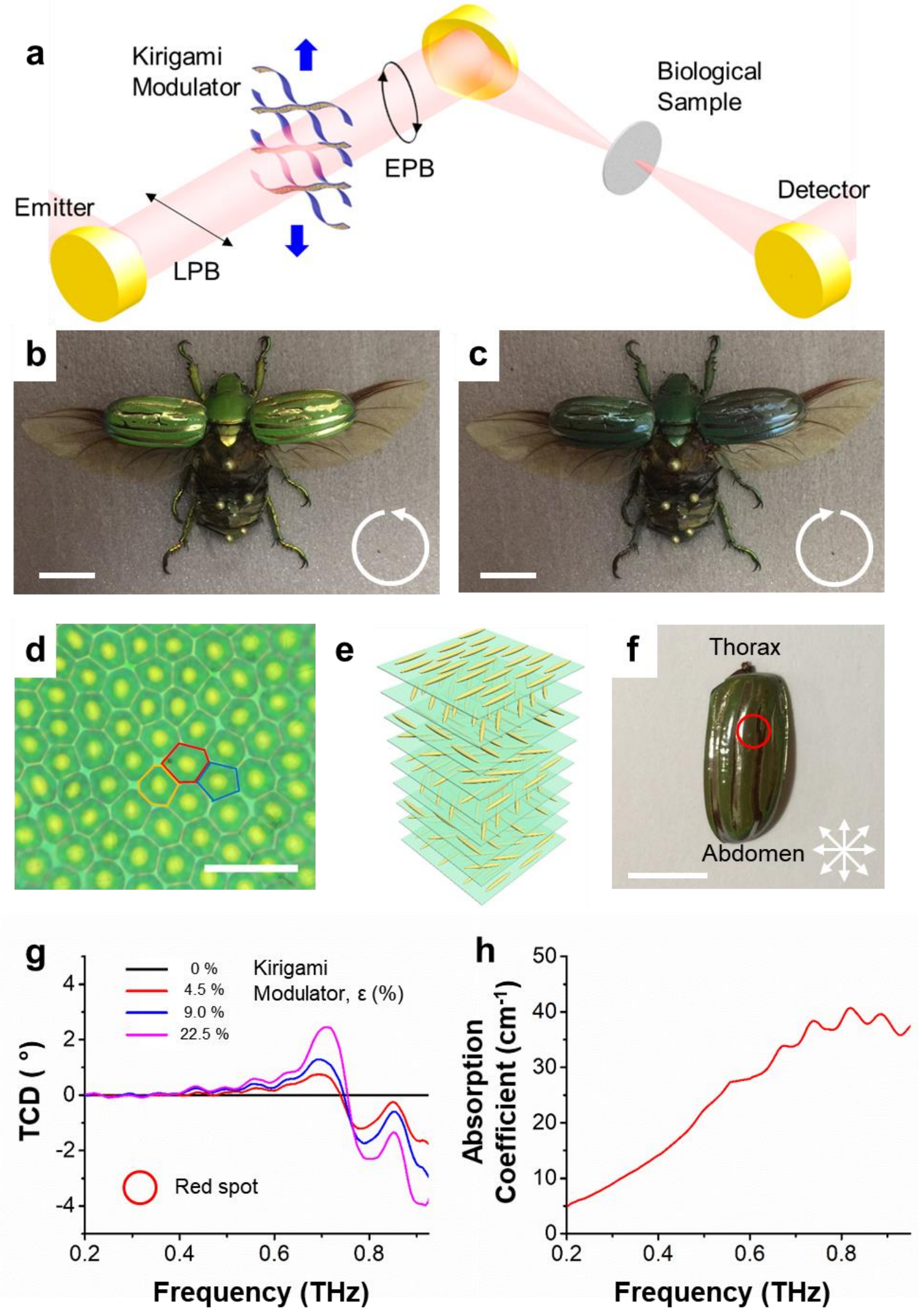
Figure 5. Measurements of TCD using kirigami chiroptical modulator. a, Schematic of TCD spectroscopy using kirigami modulator. A focused $\mathrm{THz}$ beam with $\sim 500 \mu \mathrm{m}$ spot size was used to explore biological sample. LPB and EPB indicate the linearly and elliptically polarized beam in respectively. b and c, Photographs of the beetle C.gloriosa with a left and right circular polarizer front of the camera, respectively. d, An optical microscopy image of the exoskeleton of beetle C.gloriosa. The shape of the cells is pentagonal in blue, hexagonal in red and heptagonal in orange. Scale bar is $20 \mu \mathrm{m}$. e, Schematic representation of Bouligand structure. f, Image of an elytron of C.gloriosa without polarizer. Red circle indicates the spot corresponding to the TCD measurements. g, TCD spectrum from C.gloriosa measured by kirigami modulator at four different strains (\%). h, Measured absorption coefficient of C.gloriosa. Scale bars in $\mathbf{b}, \mathbf{c}$ and $\mathbf{f}$ are $1 \mathrm{~cm}$. 


\section{REFERENCES}

1. G. Acbas, K. A. Niessen, E. H. Snell, A. G. Markelz, Optical measurements of long-range protein vibrations. Nat. Commun. 5, 3076 (2014).

2. D. F. Plusquellic, K. Siegrist, E. J. Heilweil, O. Esenturk, Applications of terahertz spectroscopy in biosystems. ChemPhysChem. 8, 2412-2431 (2007).

3. G. J. Wilmink, J. E. Grundt, Invited review article: Current state of research on biological effects of terahertz radiation. J. Infrared, Millimeter, Terahertz Waves. 32, 1074-1122 (2011).

4. J. H. Choi, M. Cho, Terahertz Chiroptical Spectroscopy of an $\alpha$-Helical Polypeptide: A Molecular Dynamics Simulation Study. J. Phys. Chem. B. 118, 12837-12843 (2014).

5. M. D. King, W. D. Buchanan, T. M. Korter, Understanding the terahertz spectra of crystalline pharmaceuticals: Terahertz spectroscopy and solid-state density functional theory study of (S)-(+)-ibuprofen and (RS)-ibuprofen. J. Pharm. Sci. 100, 1116-1129 (2011).

6. T. Kan et al., Enantiomeric switching of chiral metamaterial for terahertz polarization modulation employing vertically deformable MEMS spirals. Nat. Commun. 6, 8422 (2015).

7. N. Kanda, K. Konishi, M. Kuwata-Gonokami, Terahertz wave polarization rotation with double layered metal grating of complimentary chiral patterns. Opt. Express. 15, 1111711125 (2007).

8. S. Zhang et al., Photoinduced handedness switching in terahertz chiral metamolecules. Nat. Commun. 3, 942 (2012).

9. S. S. Oh, O. Hess, Chiral metamaterials: enhancement and control of optical activity and circular dichroism. Nano Converg. 2, 24 (2015).

10. J. Qui et al., Introducing quasi-optical terahertz circular dichroism spectroscopy. 2017 11th Int. Conf. Antenna Theory Tech. ICATT 2017. 11, 26-29 (2017).

11. S. S. Dhillon et al., The 2017 terahertz science and technology roadmap. J. Phys. D. Appl. Phys. 50 (2017)..

12. T. J. Yen et al., Terahertz Magnetic Response from Artificial Materials. Science 303, 14941496 (2004).

13. N. K. Grady et al., Terahertz Metamaterials for Linear Polarization Conversion and Anomalous Refraction. Science 340, 1304-1307 (2013).

14. S. Hunsche, M. Koch, I. Brener, M. . Nuss, THz near-field imaging. Opt. Commun. 150, 22-26 (1998).

15. W. L. Chan, J. Deibel, D. M. Mittleman, Imaging with terahertz radiation. Reports Prog. Phys. 70, 1325-1379 (2007).

16. L. Xu et al., Kirigami Nanocomposites as Wide-Angle Diffraction Gratings. ACS Nano 10, 6156-6162 (2016).

17. M. K. Blees et al., Graphene kirigami. Nature 524, 204-207 (2015).

18. M. A. Dias et al., Kirigami Actuators. Soft Matter 13, 9087 (2017).

19. Z. Liu et al., Nano-kirigami with giant optical chirality. Sci. Adv. 4, 1-9 (2018).

20. S. J. P. Callens, A. A. Zadpoor, From flat sheets to curved geometries: Origami and kirigami approaches. Mater. Today. 21, 241-264 (2018).

21. Y. Zhang et al., A mechanically driven form of Kirigami as a route to 3D mesostructures in micro/nanomembranes. Proc. Natl. Acad. Sci. U. S. A. 112, 11757-64 (2015).

22. J. Rogers, Y. Huang, O. G. Schmidt, D. H. Gracias, Origami MEMS and NEMS. MRS Bull. 
41, 123-129 (2016).

23. X. Liu et al., Metamaterials on parylene thin film substrates: Design, fabrication, and characterization at terahertz frequency. Appl. Phys. Lett. 96, 1-4 (2010).

24. J. K. Gansel et al., Gold helix photonic metamaterial as broadband circular polarizer. Science 325, 1513-5 (2009).

25. A. V Kildishev, A. Boltasseva, V. M. Shalaev, Planar photonics with metasurfaces. Science 339, 1232009 (2013).

26. N. Yu, F. Capasso, Flat optics with designer metasurfaces. Nat. Mater. 13, 139-50 (2014).

27. J. Neu, D. J. Aschaffenburg, M. R. C. Williams, C. A. Schmuttenmaer, Optimization of terahertz metamaterials for near-field sensing of Chiral substances. IEEE Trans. Terahertz Sci. Technol. 7, 755-764 (2017).

28. V. Sharma, M. Crne, J. O. Park, M. Srinivasarao, Structural origin of circularly polarized iridescence in jeweled beetles. Science 325, 449-51 (2009).

29. J. C. Weaver et al., The stomatopod dactyl club: a formidable damage-tolerant biological hammer. Science 336, 1275-80 (2012).

30. R. A. Metzler et al., Polarization-dependent imaging contrast in abalone shells. Phys. Rev. B. 77, 064110 (2008). 